\title{
Implausible Consequences of Superstrong Nonlocality
}

\author{
Wim van Dam* \\ Department of Computer Science, University of California at Santa Barbara, Santa Barbara, CA 93106-5110, USA
}

\begin{abstract}
This Letter looks at the consequences of so-called 'superstrong nonlocal correlations', which are hypothetical violations of Bell/CHSH inequalities that are stronger than quantum mechanics allows, yet weak enough to prohibit faster-than-light communication. It is shown that the existence of maximally superstrong correlated bits implies that all distributed computations can be performed with a trivial amount of communication, i.e. with one bit. If one believes that Nature does not allow such a computational 'free lunch', then the result in the Letter gives a reason why superstrong correlation are indeed not possible.
\end{abstract}

PACS numbers: 03.65.Ud, 03.65.Ta, 03.67.Hk, 03.67.Mn

Keywords: foundations of quantum mechanics, nonlocality, communication complexity

The Clauser-Horne-Shimony-Holt (CHSH) inequality [6] for classical theories gives the following upper bound on the strength of correlations between two space-like separated experiments, which can be violated by quantum mechanics. Imagine two parties Alice and Bob $(A$ and $B)$ that share a distributed system $\Phi_{A B}$. Each party can independently perform one out of two measurements on their part of the system, such that in total there are four experimental set-ups that can apply to the combined system: $\left(m_{0}^{A}, m_{0}^{B}\right),\left(m_{0}^{A}, m_{1}^{B}\right),\left(m_{1}^{A}, m_{0}^{B}\right)$ and $\left(m_{1}^{A}, m_{1}^{B}\right)$. For each measurement on each side there are two possible outcomes, which are labeled " 0 " and " 1 ". The parties repeat the experiment many times using the different settings, thus obtaining an accurate estimation of all the possible correlations between the different measurements and their outcomes. As it is understood that for each trial $A$ and $B$ always use the same state-preparation of $\Phi_{A B}$, the conditional part will be omitted when expressing the probabilities of the various outcomes. Hence, the probability that both Alice and Bob measure a "one" when they use the measurement settings $m_{0}^{A}$ and $m_{1}^{B}$ is denoted simply by $\operatorname{Prob}\left(m_{0}^{A}=1, m_{1}^{B}=1\right)$.

The main result of Bell [2] and CHSH [6] is that for any local, hidden variable theory about $\Phi_{A B}$ and the measurements $m^{A}$ and $m^{B}$, the following inequality must hold:

$$
\sum_{x, y \in\{0,1\}} \operatorname{Prob}\left(m_{x}^{A}+m_{y}^{B} \equiv x \cdot y\right) \leq 3,
$$

where we interpret the binary values as elements of 'modulo 2 calculations' such that $1+1 \equiv 0$. Quantum mechanics allows a violation of the bound of Equation 1 by

$$
\sum_{x, y \in\{0,1\}} \operatorname{Prob}\left(m_{x}^{A}+m_{y}^{B} \equiv x \cdot y\right)=2+\sqrt{2} \approx 3.41,
$$

if $A$ and $B$ use, for example, the entangled pair of quantum bits $\left|\Phi_{A B}\right\rangle=\frac{1}{\sqrt{2}}(|00\rangle+|11\rangle)$ and a suitable set of measurement projectors $m$. Besides the fact that this result proves that the theory of quantum mechanics cannot be phrased as a local theory, the more important conclusion is that the nonlocality of Nature can be verified experimentally (as has been done many times [1, 10]). This experimental aspect is the more relevant side of the matter as it is not inconceivable that in the future we will have to replace the theory of quantum mechanics by a more accurate or more general model of Nature, making the nonlocality of quantum mechanics irrelevant. But no matter its exact formulation, the succeeding theory will have to agree with our experimental results; and as the empirical data by itself rules out a local explanation, any proper future candidate theory will have to be nonlocal as well. From this perspective, which we could call 'nonlocality-without quantum physics', we should consider all possible violations of Equation 11 not just the " $2+\sqrt{2} \not \leq 3$ " violation of quantum mechanics. In this Letter we look at the plausibility of superstrong nonlocality where the nonlocal correlations are stronger than those allowed by the theory of quantum physics.

In a series of articles [12, 13, 14], Sandu Popescu and Daniel Rohrlich ask the question why Nature seems to allow a violation of the CHSH inequality with a correlation term of $2+\sqrt{2}$, but not with more. (See the article by Boris Cirel'son [5] for a proof that $2+\sqrt{2}$ is indeed the quantum mechanical limit.) They ask themselves [13]: “...Could the requirement of relativistic causality restrict the violation to $[2+\sqrt{2}]$ instead of 4?" Such a result would be great step towards a better understanding of Nature for "... If so, then nonlocality and causality would together determine the quantum violation of the CHSH inequality, and we would be closer to a proof that they determine all of quantum mechanics." Perhaps surprisingly, this turns out not to be the case. The authors prove this by constructing a toy-theory where the nonlocality Inequality 1 is surpassed by a correlation value of 4 . The non-zero probabilities of this super-nonlocal theory are simply

$$
\left.\begin{array}{l}
\operatorname{Prob}\left(m_{x}^{A}=0, m_{y}^{B}=0\right)=\frac{1}{2} \\
\operatorname{Prob}\left(m_{x}^{A}=1, m_{y}^{B}=1\right)=\frac{1}{2}
\end{array}\right\} \quad \text { if } x y \in\{00,01,10\},
$$

This leads indeed to the maximally violating correlation value

$$
\sum_{x, y \in\{0,1\}} \operatorname{Prob}\left(m_{x}^{A}+m_{y}^{B} \equiv x \cdot y\right)=4,
$$

while the randomization of the outcomes still prevents Alice or Bob from transferring information to the other party without the use of conventional communication. In fact, the probability distribution of Equation 2 is the only possible solution 
if we want to combine a correlation value of 4 with the preservation of causality.

So, if causality is still respected with the superstrong correlations of Equation 2 why does Nature not allow it? Are there any obvious first principles that forbid a violation stronger than that of quantum mechanics? When trying to answer this question in a meaningful way, it is important to remember to ignore everything one knows about quantum mechanics. As explained above, the point is to consider all possible (future) physical theories, not just the contemporary one. Cirel'son's bound already shows us that quantum mechanics is incompatible with a violation of Equation 1 that goes beyond " $2+\sqrt{2} \not \leq 3$ ", hence it is not interesting to derive a contradiction under assumptions that use features of quantum mechanics like the superposition principle, linearity, et cetera. Instead, we want to assume nothing else but a violation of Equation 1 by a value greater than $2+\sqrt{2}$.

In this Letter we look at the consequences of superstrong nonlocality for the theory of communication complexity, which describes how much communication is needed to evaluate a distributed function $f$. More specifically, consider a Boolean function $f:\{0,1\}^{n} \times\{0,1\}^{n} \rightarrow\{0,1\}$, which has as input two $n$-bit strings $\vec{x}, \vec{y} \in\{0,1\}^{n}$. If $A$ possesses the $x$ string and $B$ the $y$-string, how many bits do $A$ and $B$ have to exchange in order to determined the function value $f(\vec{x}, \vec{y})$ ? How to answer this question-which depends on the specific function and the resources of $A$ and $B$-is studied in the field of (quantum) communication complexity [11]. For certain $f$ it has been shown that quantum entanglement can reduce the amount of classical information that $A$ and $B$ need to exchange to evaluate $f$ [7], while for other functions the quantum complexity is effectively the same as the classical complexity. An example of latter is the Inner Product function $\mathrm{IP}_{n}:\{0,1\}^{n} \times\{0,1\}^{n} \rightarrow\{0,1\}$, which is defined by

$$
\operatorname{IP}_{n}\left(x_{1} \cdots x_{n}, y_{1} \cdots y_{n}\right) \equiv \sum_{i=1}^{n} x_{i} \cdot y_{i}
$$

Even if we allow $A$ and $B$ to use an unlimited amount of entangled qubits, the communication complexity will still be $n$ bits [8] (which is the maximum possible complexity as $B$ can always send $A$ all of the $n$ bits of his input $\vec{y}$, after which $A$ evaluates $f(\vec{x}, \vec{y})$ on her side).

Here it will be shown that a maximum violation of the CHSH Inequality 1 (according to the " $4 \not \leq 3$ " of Equation 3 ) leads to a situation where the notion of communication complexity is vacuous: all distributed decision problems can be solved with $100 \%$ accuracy with only one bit of communication. (Note that at least one bit needs to be communicated if we want to preserve causality.) To prove our result, we first describe a way of expressing all possible distributed functions in a standard format that coincides with the inner product problem for two parties. Then we will see how, with superstrong correlations, the IP problem (and hence all problems) can be solved with the minimal amount of one bit of communication from Bob to Alice. The results in this Letter were mentioned earlier in the Ph.D. thesis of the author [9].
Any Boolean function $f:\{0,1\}^{n} \times\{0,1\}^{n} \rightarrow\{0,1\}$ can be expressed as a multi-variable polynomial with modulo two arithmetic (where $1+1=2 \equiv 0$ ). This is most easily seen by the fact that elementary Boolean operations like AND, OR, NOT or 'equivalence' can be calculated with addition and multiplication over $\{0,1\}$ :

$$
\left\{\begin{aligned}
(x \text { AND } y) & \equiv x \cdot y, \quad(x \text { OR } y) & \equiv x+y+x \cdot y, \\
\operatorname{NOT}(x) & \equiv 1+x, \quad(x \Leftrightarrow y) & \equiv 1+x+y,
\end{aligned}\right.
$$

where the value 1 means "True" and 0 means "False". Just as any Boolean function $f:\{0,1\}^{n} \rightarrow\{0,1\}$ can be constructed from those primitives, so can $f$ be constructed from the elementary $\bmod 2$ operations " " " and ".". The 2-bit equivalence relation $\mathrm{EQ}$, for example, thus becomes

$$
\begin{aligned}
\mathrm{EQ}\left(x_{1} x_{2}, y_{1} y_{2}\right) & =\left(x_{1} \Leftrightarrow y_{1}\right) \operatorname{AND}\left(x_{2} \Leftrightarrow y_{2}\right) \\
& \equiv\left(1+x_{1}+y_{1}\right) \cdot\left(1+x_{2}+y_{2}\right) .
\end{aligned}
$$

Furthermore, we can rewrite such polynomials as a finite summation of products $f(\vec{x}, \vec{y}) \equiv \sum_{i} P_{i}(\vec{x}) \cdot Q_{i}(\vec{y})$, where $P_{i}$ are polynomials in $\vec{x} \in\{0,1\}^{n}$ and $Q_{i}$ are monomials in $\vec{y} \in\{0,1\}^{n}$. In total there are $2^{n}$ different monomials $Q_{i}(\vec{y})=\prod_{j \in S} y_{j}$ that we have to consider (one for each subset $S \subseteq\{1, \ldots, n\}$ ), hence the index $i$ in the summation can be limited to $1 \leq i \leq 2^{n}$. This gives us a way of representing the function $f$ as an inner product problem of input size $2^{n}$ :

$$
f\left(x_{1} \cdots x_{n}, y_{1} \cdots y_{n}\right) \equiv \sum_{i=1}^{2^{n}} P_{i}(\vec{x}) \cdot Q_{i}(\vec{y}),
$$

with $\vec{x}, \vec{y} \in\{0,1\}^{n}$. For the example of the 2-bit equality function EQ this is shown by

$$
\begin{aligned}
\mathrm{EQ}\left(x_{1} x_{2}, y_{1} y_{2}\right) \equiv & \left(1+x_{1}+y_{1}\right) \cdot\left(1+x_{2}+y_{2}\right) \\
\equiv & \left(1+x_{1}+x_{2}+x_{1} x_{2}\right) \cdot 1+1 \cdot y_{1} y_{2} \\
& +\left(1+x_{2}\right) \cdot y_{1}+\left(1+x_{1}\right) \cdot y_{2} \\
\equiv & \sum_{i=1}^{4} P_{i}\left(x_{1}, x_{2}\right) \cdot Q_{i}\left(y_{1}, y_{2}\right) .
\end{aligned}
$$

We can view this as an inner product problem because all the bit values $P_{i}(\vec{x})$ are known to Alice and all the values $Q_{i}(\vec{y})$ are known to Bob without the need for any communication between them. Hence, if $A$ and $B$ are able to compute the IP function for input sizes of $2^{n}$ with one bit of communication, then they are also able to calculate any decision problem $f:\{0,1\}^{n} \times\{0,1\}^{n} \rightarrow\{0,1\}$ with a single bit of information exchange. Next we will see that this indeed possible with a maximum violation of the CHSH inequality.

Assume a model of Nature where the probabilities of Equations 2 and 3 are applicable, and hence where the correlation

$$
\operatorname{Prob}\left(m_{x}^{A}+m_{y}^{B} \equiv x \cdot y\right)=1
$$

holds for all $x, y \in\{0,1\}$. In such a world Alice and Bob (with input bits $x$ and $y$ ) can perform two separated measurements on their super-correlated states that yield the outcomes $\alpha$ and $\beta$ obeying $\alpha+\beta \equiv x \cdot y$. From this it follows 
that in the case of the inner product function $\mathrm{IP}_{N}$ on strings of length $N$, Alice and Bob can perform $N$ measurements on $N$ super-correlated particle pairs in order to obtain - without any communication-a collection of bit values $\alpha_{i}$ and $\beta_{i}$, with $\alpha_{i}+\beta_{i} \equiv x_{i} \cdot y_{i}$ for every $1 \leq i \leq N$. The commutativity of addition (modulo two) allows the following regrouping of the bits by the two separated sides of the communication protocol:

$$
\begin{aligned}
\operatorname{IP}_{N}\left(x_{1} \cdots x_{N}, y_{1} \cdots y_{N}\right) & \equiv \sum_{i=1}^{N} x_{i} \cdot y_{i} \\
& \equiv \sum_{i=1}^{N}\left(\alpha_{i}+\beta_{i}\right) \\
& \equiv \underbrace{\left(\sum_{i=1}^{N} \alpha_{i}\right)}_{\text {Alice's side }}+\underbrace{\left(\sum_{i=1}^{N} \beta_{i}\right)}_{\text {Bob's side }} .
\end{aligned}
$$

Because Bob can construct and add his $\beta_{i}$ values without requiring any information from Alice, he can therefore compute the value $b \equiv \sum_{i} \beta_{i}$ by himself and broadcast this single bit to Alice. She, on her part, creates the $\alpha_{i}$ values and finishes the protocol with the errorless conclusion $\operatorname{IP}(x, y) \equiv b+\sum_{i} \alpha_{i}$.

We just saw how the IP function has a communication complexity of one bit for every finite input size $N$ in the setting of superstrong correlations. Hence, we can apply the reduction shown earlier to reach the result that any distributed decision problem $f(\vec{x}, \vec{y})$ can be exactly computed with a single bit of communication. Equation 4 tells us that we can rewrite the function $f$ to $f(\vec{x}, \vec{y}) \equiv \sum_{i} P_{i}(\vec{x}) \cdot Q_{i}(\vec{y})$. As Bob can compute all the $Q_{i}$ values by himself, he and Alice can also remotely and independently create the $\alpha_{i}$ and $\beta_{i}$ values such that $\alpha_{i}+\beta_{i} \equiv P_{i}(x) \cdot Q_{i}(y)$ for all $1 \leq i \leq 2^{n}$. After the appropriate regrouping of the sum, Equation 4 then becomes

$$
f\left(x_{1} \cdots x_{n}, y_{1} \cdots y_{n}\right) \equiv \underbrace{\left(\sum_{i=1}^{2^{n}} \alpha_{i}\right)}_{\text {Alice's side }}+\underbrace{\left(\sum_{i=1}^{2^{n}} \beta_{i}\right)}_{\text {Bob's side }} .
$$

It should now be clear that Bob can compute the bit $b \equiv \sum_{i} \beta_{i}$ by himself and then communicate it to Alice who, just as for the IP function, concludes with $f(\vec{x}, \vec{y}) \equiv b+\sum_{i} \alpha_{i}$.

This finishes the proof that with the help of the superstrong correlations of Equation 2 any distributed function can be decided on Alice's side without error after only one bit of communication from Bob. It is true that in this protocol the amount of resources (the super-correlated states) can grow exponentially with the input size $n$ but this does not effect the conclusion that the communication complexity-after the inputs are distributed-is minimal.

We can now rephrase our original question as: why would Nature not allow super-efficient distributed computing? It is not clear if there is a convincing answer to this, as it does not seem to conflict with any physical intuition. However, trivial communication complexity does prohibit the existence of an intrinsic 'complexity' for distributed tasks. Even though we need an exponential amount of prior superstrong nonlocality (as is indeed sometimes the case), the solution of all possible distributed functions with a single bit of communication does contradict our experiences that certain computational tasks are harder than other ones. Similar as in computability theory, there is a hierarchy of different complexity classes of communication problems [3]. Such hierarchies are at the core of theoretical computer science, and their absence-as happened here by assuming superstrong correlations-goes squarely against the worldview and experience of probably all researchers in the field of complexity theory.

If we accept the absence of intrinsic complexity as an argument against superstrong correlations, then it is natural to wonder if we can obtain similar results for correlations that are less strong than those of Equation 3 It is tempting to speculate that a more detailed analysis would reveal that the $2+\sqrt{2}$ of quantum mechanics is a critical value that separates trivial from nontrivial communication complexity, which would give an argument for Cirel'son's nonlocality bound without referring to quantum mechanics. Recent work by Buhrman et al. [4] shows that trivial communication complexity can indeed be achieved with a correlation value strictly less than 4 . Because there is a tight connection between the communication complexity of distributed functions and the depth of circuits for these problems [11] it also possible to consider the implications of superstrong nonlocality for computational complexity.

* Email: vandam@ cs.ucsb.edu

[1] A. Aspect, J.Dalibard, and G. Roger, "Experimental test of Bell's inequalities using time-varying analyzers", Physical Review Letters, 49:1804-1807, 1982.

[2] J.S. Bell, "On the Einstein-Podolsky-Rosen paradox," Physics, 1:195-200, 1964.

[3] L. Babai, P.G. Frankl, and J. Simon, "Complexity classes in communication complexity theory", Proceedings of the 27th IEEE Symposium on Foundations of Computer Science, pages 337-347, IEEE Computer Society Press, 1986.

[4] H. Buhrman, R. Cleve, N. Linden, and F. Unger, in preparation.

[5] B.S. Cirel'son, "Quantum generalizations of Bell's inequality", Letter in Mathematical Physics, 4:93-100, 1980.

[6] J.F. Clauser, M.A. Horne, A. Shimony, and R.A. Holt, "Proposed experiment to test local hidden-variable theories", Physical Review Letters, 23:880-884, 1969.

[7] R. Cleve and H. Buhrman, "Substituting quantum entanglement for communication", Physical Review A, 56(2):1201-1204, 1997; arXiv:quant-ph/9704026

[8] R. Cleve, W. van Dam, M. Nielsen, and A. Tapp, "Quantum Entanglement and the Communication Complexity of the Inner Product Function", Lecture Notes in Computer Science, Volume 1509, pp. 71-74, 1998; arXiv:quant-ph/9708019

[9] W. van Dam, Chapter 9 in "Nonlocality \& Communication Complexity", D.Phil. thesis, University of Oxford, Department of Physics, 2000

[10] S.J.Freedman and J.F.Clauser, "Experimental test of local hidden-variable theories", Physical Review Letters, 28:938$941,1972$. 
[11] E. Kushilevitz and N. Nisan, Communication Complexity, Cambride University Press, Cambridge, United Kingdom, 1997.

[12] S. Popescu and D. Rohrlich, "Quantum nonlocality as an axiom”, Foundations of Physics, 24(3):379-385, 1994.

[13] S. Popescu and D. Rohrlich, "The relativistic EPR argument", in R.S.Cohen, M. Horne, and J.J. Stachel, editors, Potentiality, entanglement and passion-at-a-distance: quantum mechanical studies for Abner Shimony, Volume Two, volume 194 of Boston studies in the philosophy of science, Kluwer Academic Publishers, Dordrecht, The Netherlands, 1997;
arXiv:quant-ph/9605004 as "Action and Passion at a Distance: An Essay in Honor of Professer Abner Shimony".

[14] D. Rohrlich and S. Popescu, "Nonlocality as an axiom for quantum theory", in A. Mann and M. Revzen, editors, The dilemma of Einstein, Podolsky and Rosen, 60 years later: International symposium in honour of Nathan Rosen, volume 12 of Annals of the Israel Physical Society, chapter 16, Israel Physical Society, Haifa, Israel, 1996; arXiv:quant-ph/9508009 